\title{
Two peculiar new Orfelia Costa species from Georgia (Diptera: Keroplatidae)
}

\section{Olavi Kurina \& Siiri Jürgenstein}

\begin{abstract}
Kurina, O. \& Jürgenstein, S. 2013: Two peculiar new Orfelia Costa species from Georgia (Diptera: Keroplatidae). — Entomol. Fennica 24: 21-27.

Two new Keroplatidae species, Orfelia georgica sp. n. and Orfelia trifida sp. n., are described on the basis of male material collected from Georgia (Caucasus). The two species, representing the first records of the genus in the country, are photographed, their terminalia illustrated and systematics discussed.

O. Kurina \& S. Jürgenstein, Institute of Agricultural and Environmental Sciences, Estonian University of Life Sciences, Riia st 181, 51014 Tartu, Estonia; Corresponding author's e-mail: olavi.kurina@emu.ee
\end{abstract}

Received 21 August 2012, accepted 29 October 2012

\section{Introduction}

The genus Orfelia Costa, 1857, the nominal genus of Orfeliini is placed in the subfamily Keroplatinae of family Keroplatidae (Søli et al. 2000, Evenhuis 2006). Within the tribial limits, the genus is defined by the following combination of characters: short mouthparts, bare laterotergite, setose mediotergite, bare medial and cubital veins and regular rows of tibial setae (e.g. Søli et al. 2000).

Altogether 42 species have been recorded up to the present, viz. 23 species from the Palaearctic region including 14 from Europe, 11 from the Nearctic region, 7 from the Oriental and 2 from the Neotropical region (Evenhuis 2006). One species, O. discoloria (Meigen, 1818), is known to have a Holarctic distribution. No species have yet been reported from the Afrotropical and Australasian regions. By unidentified specimens, the genus has been known also from Baltic amber (Evenhuis 1994).

There is no synopsis to cover all known species, while the most comprehensive keys are published for European and Palaearctic species by Hutson et al. (1980) and Zaitzev (1994), respec- tively. However, the keys include only a selection of the actual species in these areas, hence, additional literature including descriptions of single species (e.g. Zaitzev \& Menzel 1996, Bechev 2002) should be consulted.

Species of Keroplatidae are rather superficially known in Transcaucasia (i.e. Armenia, Azerbaijan and Georgia). Nine species have been recorded in the area while only one species (Isoneuromyia semirufa (Meig.)) has been previously reported from Georgia (Zaitzev 1994). Five additional keroplatid species are known from neighbouring North-, West- and Central-Caucasus (see Joost \& Plassmann 1976, 1979, 1985 , 1992). Only two Orfelia species, O. matilei Zaitzev, 1994 and O. unicolor (Staeger, 1840), are recorded from the whole Caucasian region up to the present (Zaitzev 1994).

The aim of the current communication is to describe and illustrate two new Orfelia species from Georgia and discuss their systematics.

\section{Material and methods}

All material has been collected by O.K. during a 




Fig. 1. - a, b. Collecting locality in surroundings of Marelisi village southeast of Surami, Georgia. - c. Habitus of Orfelia georgica sp. n. - d. Habitus of Orfelia trifida sp. n., terminalia detached. Scale bar $=1 \mathrm{~mm}$.

recent expedition to Georgia. The collecting locality, surroundings of Marelisi village near Borjomi-Kharagauli National Park, is characterised by hornbeams, beech, chestnut and lime dominated deciduous forest along slopes of hills (Fig. 1a) including many small and narrow ravines (Fig. 1b).

All material was collected by sweep netting and was initially preserved in $70 \%$ ethanol. After study, some of the specimens were mounted from alcohol, using a chemical method described by Vockeroth (1966), and double-mounted. For a detailed study and illustration of terminalia, they were detached and treated by the method described by Kurina (2003), before being inserted into glycerine. After examination, terminalia were stored in the glycerine medium in a special plastic microvial attached to the same pin as the mounted specimen.

Illustrations of the terminalia were prepared using a U-DA drawing tube attached to a compound microscope Olympus CX31. Habitus photos (Fig. 1c, d) were made in ethanol medium, using a Canon 7D camera in combination with Canon MP-E65 (F2.8 1-5×) lens (see also Kurina 2008). Male terminalia are figured in three different positions: ventrally, laterally and dorsally, while tergite 9 was detached and figured separately. Adobe Photoshop CS5 software was used to add shading to the figures.

Morphological terminology follows Søli (1997). The measurements are given as the range of measured specimens followed by the mean value when at least 5 specimens were measured, while the measurements from the holotype are given in square brackets. The ratios of the three apical palpal segments are given as $3^{\text {rd }}: 4^{\text {th }}: 5^{\text {th }}$. All material is deposited in the insect collection in IZBE, Institute of Agricultural and Environmental Sciences, Estonian University of Life Sci- 
Fig. 2. Orfelia georgica sp. n., male terminalia. - a. Ventral view. - b. Lateral view. - c. Dorsal view, tergite 9 detached. - d. Tergite 9. Scale bar $=0.1$ $\mathrm{mm}$.
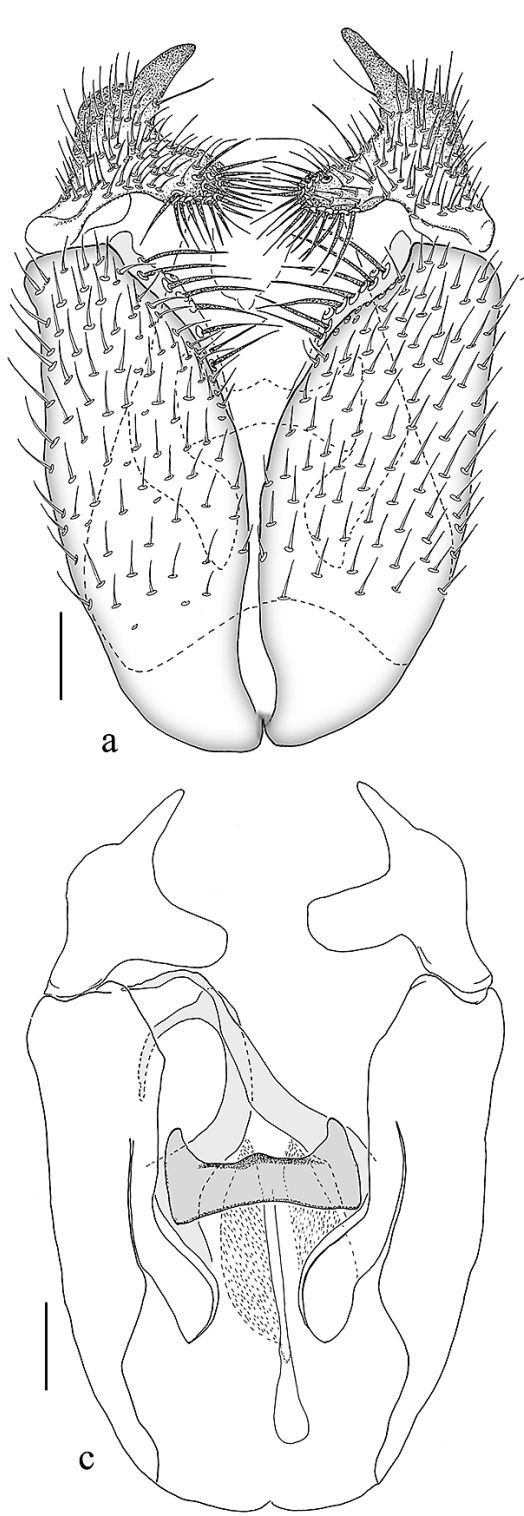


ences (former Institute of Zoology and Botany), Tartu, Estonia.

\section{Systematics}

\subsection{Orfelia georgica sp. n. (Figs. 1c, 2a-d)}

Type material. Holotype. ${ }^{7}$, GEORGIA, Marelisi southeast of Surami, 41 ${ }^{\circ} 56^{\prime} 48.0^{\prime \prime} \mathrm{N} 043^{\circ} 17^{\prime}$ 03.3"E, $448 \mathrm{~m}$ a.s.1., sweeping 20.V.2012, O. Kurina leg. [dry mounted from alcohol, IZBE].
Paratypes. ${ }^{\lambda}$, same as holotype except $41^{\circ} 56^{\prime}$ 22.7'N $043^{\circ} 16$ '37.3'E, $693 \mathrm{~m}$ a.s.l. [on pin, IZBE]; $10 \overbrace{}^{\top} \mathrm{O}^{\lambda}$, same as holotype except $41^{\circ} 57^{\prime}$ 56.0'N 043 ${ }^{\circ} 17^{\prime} 20.7$ 'E, $412 \mathrm{~m}$ a.s.l., sweeping 19.V.2012 [dry mounted from alcohol, IZBE].

Additional material studied. $5 \hat{\partial} \hat{\partial}$, same as holotype except $41^{\circ} 57^{\prime} 56.0^{\prime \prime} \mathrm{N} 043^{\circ} 17^{\prime} 20.7^{\prime}$ 'E, $412 \mathrm{~m}$ a.s.1., sweeping 19.V.2012 [in alcohol, IZBE].

Description. Male. Body length 5.49-6.50, $5.97[6.03] \mathrm{mm}$, wing length 4.76-5.16, 4.87 [4.82] $\mathrm{mm}(n=5)$. 
Head. Blackish brown with dark setae. Three ocelli in shallow triangular arrangement, median ocellus smaller than laterals. Clypeus rectangular, brown, with dark setae on lower part. Mouthparts yellow. Maxillary palpus yellow, with dark setae. Ratios of three apical palpal segments 1.0:0.831.00, 0.93 [1.00]:1.47-1.93, 1.67 [1.93]; penultimate segment $2.14-2.67,2.46$ [2.14] times as long as wide, last segment 6.00-7.75, 6.85 [7.25] times as long as wide. Antenna dark brown with scape, pedicel and basal part of first flagellomere lighter to yellowish. Flagellomeres quadrate with dark short setae, median flagellomere about 0.9 times as long as broad, apical flagellomere elongated, 2.25-2.75, 2.51 [2.25] times as long as broad basally, with clearly outlined apical papilla.

Thorax. Mesonotum dark brown with yellowish humeri; all setae black. Scutellum brown, basally somewhat yellowish, with marginal dark short setae not arranged to distinct pairs. Lateral parts of thorax bicoloured: antepronotum, anepisternum, aneprimeron and preepisternum 2 light brown; proepisternum, laterotergite and mediotergite darker but lighter than mesonotum. Antepronotum, laterotergite and mediotergite yellowish on upper part; preepisternum 2 whitish pale on lower part. Antepronotum and proepisternum with short dark setae, mediotergite with dark setae on hind part. Other thoracic sclerites bare. Halter pale yellow with dark short setae on stem and on anterior two third of knob. Apex of knob bare.

Legs yellow with all setae blackish. Tarsal segments seem darker because of dense setae. Ratio of femur to tibia for fore, mid and hind legs: $0.80-0.86,0.82$ [0.80]; 0.68-0.79, 0.72 [0.72]; $0.63-0.71,0.69$ [0.63]. Ratio of tibia to basitarsus for fore, mid and hind legs: 1.04-1.13, 1,08 [1.04]; 1.17-1.25, 1.20 [1.17]; 1.20-1.27, 1.23 [1.20].

Wing with yellowish tinge along anterior margin and with preapical, gradually paler brownish band from the apex of R5 to the M2 or occasionally and very faintly to wing margin, leaving the apex of wing hyaline. Veins light brown, with R1, R4, R5 and CuA2 darker, M2 basally obsolete. R5 setose on both side, R1 setose dorsally, $\mathrm{CuA} 2$ with 7-10 dorsal setae before and at level of bm-cu. Otherwise all veins bare. Sc ends in costa clearly before base of Rs. R4 0.19-
$0.26,0.23[0.26]$ times as long as apical portion of $\mathrm{R} 5$. $\mathrm{R} 4$ ends in costa 0.3 distance from R1 to R5. Costa reaches about 0.3 distance from $\mathrm{R} 5$ to $\mathrm{M} 1$. $\mathrm{m}$-stem about 1.2 as long as basal portion of R5. Branches of medial fork apically parallel. Branches of cubital fork gradually divergent towards wing margin.

Abdomen. Dorsoventrally slightly compressed, brown. First segment with yellow posterolateral spots, II to VI segments with yellow bands at posterior margin. All setosity on abdomen brownish black. Terminalia brown with gonocoxite slightly yellowish apically. Tergite 9 tapering with shallow anterior incision. Gonocoxite with strong marginal bristles bearing internal flange at ventromesal margin. Gonostylus bifid: basal lobe with aggregation of strong apical mesally directed bristles, apical lobe almost bare with 2-3 short setae medially.

Female. Unknown.

Biology. Unknown.

Etymology. The name refers to the Georgian type locality.

\subsection{Orfelia trifida sp. n. (Figs. 1d, 3a-d)}

Type material. Holotype. $\hat{\jmath}$, GEORGIA, Marelisi southeast of Surami, 41 ${ }^{\circ} 57^{\prime} 03.6^{\prime \prime} \mathrm{N}$ $043^{\circ} 17^{\prime} 00.7^{\prime}$ E, $434 \mathrm{~m}$ a.s.1., sweeping 18.V. 2012, O. Kurina leg. [dry mounted from alcohol, IZBE].

Description. Male $(n=1)$. Body length [5.4 $\mathrm{mm}$ ], wing length [4.62 $\mathrm{mm}$ ].

Head. Blackish brown with dark setae. Three ocelli in shallow triangular arrangement, median ocellus smaller than laterals. Clypeus rectangular, dark brown, with dark setae on lower part. Mouthparts yellow. Maxillary palpus yellow, with dark setae. Ratios of three apical palpal segments 1.0:1.13:1.8, penultimate segment 2.8 times as long as wide, last segment 9 times as long as wide. Antenna dark brown with basal part of first flagellomere yellowish. Flagellomeres quadrate with dark short setae, median flagellomere about 0.9 times as long as broad, apical flagellomere elongated, 1.9 times as long as broad basally, with clearly outlined apical papilla.

Thorax. Mesonotum dark brown with setae dark. Scutellum brown, basally somewhat yellowish, with marginal dark short setae not ar- 


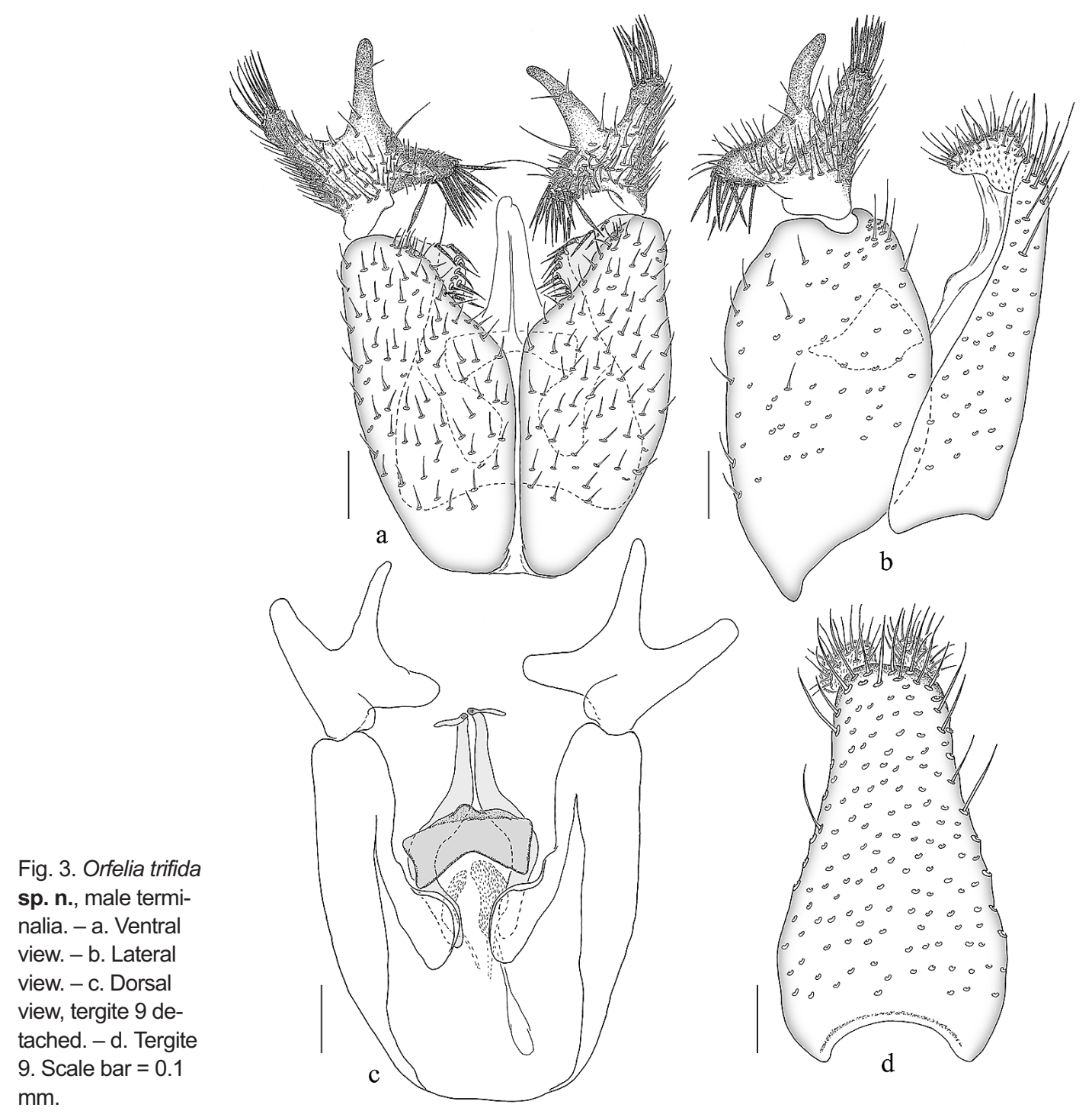

ranged to distinct pairs. Lateral parts of thorax brown, somewhat lighter than mesonotum. Mediotergite yellowish on upper part. Antepronotum and proepisternum with short dark setae, mediotergite with dark setae on hind part. Other thoracic sclerites bare. Halter pale yellow with dark short setae on stem and on anterior two third of knob. Apex of knob bare.

Legs yellow with all setae blackish. Tarsal segments seem darker because of dense setae. Ratio of femur to tibia for fore, mid and hind legs: $0.86 ; 0.75 ; 0.73$. Ratio of tibia to basitarsus for fore, mid and hind legs: $1.05 ; 1.23 ; 1.21$.

Wing with yellowish tinge along anterior margin and with preapical, gradually paler brownish band from the apex of R5 to the middle of medial fork, leaving the apex of wing hyaline. Veins light brown, with R1, R4, R5 and $\mathrm{CuA} 2$ darker, M2 basally obsolete. R5 setose on both side, R1 setose dorsally, $\mathrm{CuA} 2$ with 5-6 dorsal setae at level of bm-cu. Otherwise all veins bare. Sc ends in costa before base of Rs. R4 0.2 times as long as R5. R4 ends in costa 0.3 distance from R1 to R5. Costa reaches 0.2 distance from R5 to M1. $\mathrm{m}$-stem about as long as basal portion of R5. Branches of medial fork apically slightly convergent. Branches of cubital fork gradually divergent towards wing margin. 
Abdomen. Dorsoventrally compressed. First segment entirely brownish, succeeding segments with yellowish posterior bands which are more clearly outlined on tergites laterally and on sternites. All setosity on abdomen brownish black. Tergite 9 tapering with shallow anterior incision. Gonocoxite with strong marginal bristles bearing internal flange at ventromesal margin. Gonostylus trifid: basal and lateral lobes with aggregation of strong apical bristles, medial lobe almost bare with 3-4 short setae medially.

Female. Unknown.

Biology. Unknown.

Etymology. The name is Latin - trifida, divided into three or consisting of three parts, referring to the trifid shape of gonostylus of male terminalia: adjective.

\section{Discussion}

The Palaearctic Orfelia species can be divided into two groups by the coloration of the mesonotum (entirely brown to blackish versus yellow to ochrous, with or without dark longitudinal stripes) and the structure of the gonostylus (single versus basally divided) (cf. Zaitzev 1994, Chandler et al. 2006). Both new species belong to the group having a dark brown mesonotum and a single gonostylus of the male terminalia, sharing the combination of characters with three other Palaearctic species, viz. O. bezzii (Strobl, 1910), O. bicolor (Macquart, 1826) and O. nemoralis (Meigen, 1818). The first two of these species have the gonostylus without lobes (cf. Chandler et al. 2006: fig. 19, Chandler 2001: fig. 6) while the latter has a basally undivided single gonostylys with two lobes (cf. Hutson et al. 1980: fig. 144, Zaitzev 1994: fig. 40-3). By the structure of the gonostylus, a Japanese species, O. angulata (Sasakawa, 1994) having otherwise a yellow mesonotum, resembles O. nemoralis (cf. Sasakawa 1994: fig. 1). Orfelia georgica $\mathbf{s p .}$. has, similarly to these two species, a bifid single gonostylus: the basal lobe bears strong apical bristles while the apical lobe is sclerotized and almost bare. In contrast, $O$. nemoralis and $O$. angulata have the apical lobe of gonostylus setose and stockier. Orfelia trifida sp. n. has a trifid gonostylus that is unique within the genus Orfelia, including an additional lateral setose lobe with strong apical bristles. Both new species have, similar to $O$. angulata, marginal bristles bearing an internal flange at the ventroapical margin of the gonocoxite mesally. According to Zaitzev's key (1994), the new species run to couple 4 because of the mainly brown thoracic coloration and the elongated palpal segments. However, in contrast to the species included in this couplet, both new species lack pointed outgrowths on the dorsoapical margin of the gonocoxites.

Acknowledgements. The study was supported by the grants 9174 and 8583 of the Estonian Science Foundation and by target financing project SF0170160s08. We are grateful to Prof. T. Tammaru (Tartu, Estonia) for organising the collecting trip to Georgia in May 2012, to Dr. G. Japoshvili (Tbilisi, Georgia) for help during the fieldwork in Georgia and to Dr. J. Kjærandsen (Lund, Sweden) for comments on the species. We thank Dr. N. Evenhuis (BMH, Honolulu, Hawaii) for reading and critical perusal of the manuscript.

\section{References}

Bechev, D. N. 2002: Orfelia gruevi spec. nov. from Bulgaria (Diptera: Sciaroidea: Keroplatidae). - Acta Entomologica Slovenica 10: 199-201.

Chandler, P. J. 2001: Fungus gnats (Diptera: Sciaroidea) new to Britain. - British Journal of Entomology and natural History 13: 215-243.

Chandler, P. J., Bechev, D. N., \& Caspers, N. 2006: The fungus gnats (Diptera: Bolitophilidae, Diadocidiidae, Ditomyiidae, Keroplatidae and Mycetophilidae) of Greece, its islands and Cyprus. — Studia Dipterologica 12: 255-314.

Evenhuis, N. L. 1994: Catalogue of the fossil flies of the world (Insecta: Diptera). — Backhuys Publishers, Leiden. 600 pp.

Evenhuis, N. L. 2006: Catalog of the Keroplatidae of the World (Insecta: Diptera). - Bishop Museum Bulletin in Entomology 13. Honolulu, Bishop Museum Press. $178 \mathrm{pp}$.

Hutson, A. M., Ackland, D. M., \& Kidd, L. N. 1980: Mycetophilidae (Bolitophilinae, Ditomyiinae, Diadocidiinae, Keroplatinae, Sciophilinae and Manotinae) (Diptera, Nematocera). - Handbooks for the Identification of British Insects 9, 3. Royal Entomological Society of London. $111 \mathrm{pp}$.

Joost, W. \& Plassmann, E. 1976: Mycetophiliden aus dem Zentralkaukasus (Diptera: Mycetophilidae). - Senckenbergiana biologica 57: 67-68.

Joost, W. \& Plassmann, E. 1979: Zur Pilzmücken-Fauna im West-Kaukasus (Insecta: Diptera: Mycetophilidae). - Senckenbergiana biologica 59(1978): 369 370 .

Joost, W. \& Plassmann, E. 1985: Pilzmücken aus dem 
Kaukasus-Gebiet (UdSSR) (Insecta, Diptera, Mycetophilidae). - Faunistische Abhandlungen 12: 137139.

Joost, W. \& Plassmann, E. 1992: Beitrag zur Kenntnis kaukasischer Pilzmücken. (Insecta, Diptera: Mycetophilidae). - Faunistische Abhandlungen 18: 209-211.

Kurina, O. 2003: Notes on the Palaearctic species of the genus Polylepta Winnertz (Diptera: Mycetophilidae) with a new synonymizaton. - Entomologica Fennica 14: 91-97.

Kurina, O. 2008: Cluzobra matilei sp. n. from French Guyana, with notes on congeners (Diptera: Mycetophilidae). - Zootaxa 1874: 63-68.

Sasakawa, M. 1994: Fungus gnats associated with flowers of the genus Arisaema (Araceae). Part 2. Keroplatidae and Mycetophilidae (Diptera). - Transactions of the Shikoku Entomological Society 20: 293-306.
Søli, G. E. E. 1997: On the morphology and phylogeny of Mycetophilidae, with a revision of Coelosia Winnertz (Diptera, Sciaroidea). — Entomologica Scandinavica Supplement 50: 1-139.

Søli, G. E. E., Vockeroth, R. J. \& Matile, L. 2000: A. 4. Families of Sciaroidea. — In: Papp, L. \& Darvas, B. (eds.), Contribution to a Manual of Palaearctic Diptera. Appendix: 49-92. Science Herald. Budapest. 604 pp.

Vockeroth, J. R. 1966: A method of mounting insects from alcohol. - The Canadian Entomologist 98: 69-70.

Zaitzev, A. I. 1994: Fungus gnats of the fauna of Russia and adjacent regions. Part 1. Moscow. 288 pp. [In Russian.]

Zaitzev, A. I., \& Menzel, F. 1996: New data on the fungus gnats from the Russian Far East (Diptera: Sciaroidea). - Beiträge zur Entomologie 46: 159-167. 